\title{
The effect of metallic substrates on the optical properties of monolayer $\mathrm{MoSe}_{2}$
}

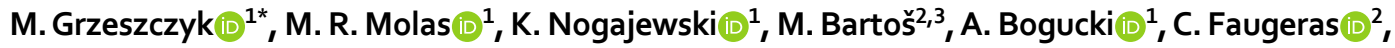 \\ P. Kossacki@ ${ }^{1}$, A. Babiński $\mathbb{D}^{1} \&$ M. Potemski $\mathbb{D}^{1,2}$
}

Atomically thin materials, like semiconducting transition metal dichalcogenides (S-TMDs), are highly sensitive to the environment. This opens up an opportunity to externally control their properties by changing their surroundings. Photoluminescence and reflectance contrast techniques are employed to investigate the effect of metallic substrates on optical properties of $\mathrm{MoSe}_{2}$ monolayer (ML). The optical spectra of $\mathrm{MoSe}_{2}$ MLs deposited on $\mathrm{Pt}, \mathrm{Au}, \mathrm{Mo}$ and $\mathrm{Zr}$ have distinctive metal-related lineshapes. In particular, a substantial variation in the intensity ratio and the energy separation between a negative trion and a neutral exciton is observed. It is shown that using metals as substrates affects the doping of S-TMD MLs. The explanation of the effect involves the Schottky barrier formation at the interface between the $\mathrm{MoSe}_{2} \mathrm{ML}$ and the metallic substrates. The alignment of energy levels at the metal/semiconductor junction allows for the transfer of charge carriers between them. We argue that a proper selection of metallic substrates can be a way to inject appropriate types of carriers into the respective bands of S-TMDs.

Out-of-plane quantum confinement in monolayers (MLs) of semiconducting transition metal dichalcogenides (S-TMDs), as well as their large in-plane effective masses of electrons and holes contribute to strong Coulomb interactions between charge carriers, which is reflected in large exciton binding energies ${ }^{1,2}$. Due to the nature of those materials, their electronic and optical properties are highly sensitive to their surroundings. This can be used as a non-invasive way to influence the screening of electron-hole Coulomb interaction in S-TMDs MLs ${ }^{3-7}$. On the other hand, the electronic properties of atomically thin layers can be locally altered by metals, which are contacted with the samples ${ }^{8-10}$. In consequence, using metals as substrates may affect the doping of S-TMD MLs due to the alignment of energy bands at the metal/semiconductor junctions. A selection of suitable substrates can be a way to inject appropriate types of carriers into the respective bands of S-TMDs. Better understanding of the role of interfaces and doping processes is important for future applications of thin S-TMD layers in a variety of modern electronic devices (field-effect transistors ${ }^{11}$, sensors ${ }^{12}$, spintronic ${ }^{13}$ and valleytronic circuits ${ }^{14}$ etc.) since all of them incorporate metallic contacts.

We study the effect of metallic substrate on optical properties of $\mathrm{MoSe}_{2} \mathrm{ML}$. The ground exciton state of the $\mathrm{MoSe}_{2} \mathrm{ML}$ is bright ${ }^{15}$ and the corresponding emission spectrum comprises two peaks related to neutral and charged excitons ${ }^{16,17}$. Metals, on top of which the $\mathrm{MoSe}_{2}$ flakes were transferred, were chosen based on their fundamental physical properties: electrical and thermal conductance, work functions, and chemical stability. Platinum $(\mathrm{Pt})$ and gold $(\mathrm{Au})$ are often used as high-work-function electrical contacts (the work functions of $\mathrm{Pt}$ and $\mathrm{Au}$ are equal to $5.64 \mathrm{eV}^{18}$ and $5.1 \mathrm{eV}^{19}$, respectively). When connected to monolayer $\mathrm{MoSe}_{2}$ they are expected to form p-type Schottky barriers. The opposite should be observed for zirconium (Zr), characterised by low work function (equal to $4.05 \mathrm{eV}^{19}$ ) and supposed to result in n-type Schottky contacts. We also consider molybdenum (Mo) that should form strong orbital overlaps with materials comprising the same element, in particular, $\mathrm{MoSe}_{2}$. A diagram representation of the energy structure of $\mathrm{ML} \mathrm{MoSe} \mathrm{M}_{2}$ metal junctions under study is shown in Fig. 1(a). The investigated samples are schematically illustrated in Fig. 1(b).

\footnotetext{
${ }^{1}$ Institute of Experimental Physics, Faculty of Physics, University of Warsaw, ul. Pasteura 5, 02-093, Warsaw, Poland. ${ }^{2}$ Laboratoire National des Champs Magnétiques Intenses, CNRS-UGA-UPS-INSA-EMFL, 25, avenue des Martyrs, 38042 , Grenoble, France. ${ }^{3}$ Central European Institute of Technology, Brno University of Technology, Purkyňova 656/123, 612 00, Brno, Czech Republic. *email: magdalena.grzeszczyk@fuw.edu.pl
} 
(a)

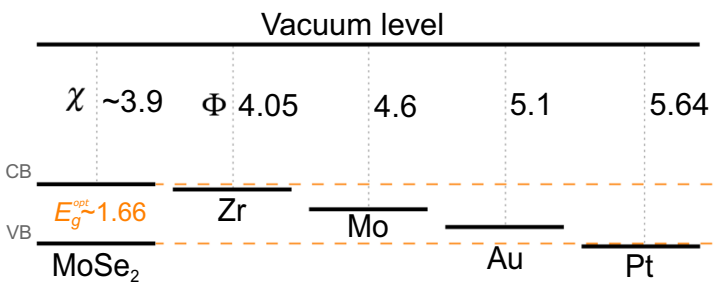

(c)

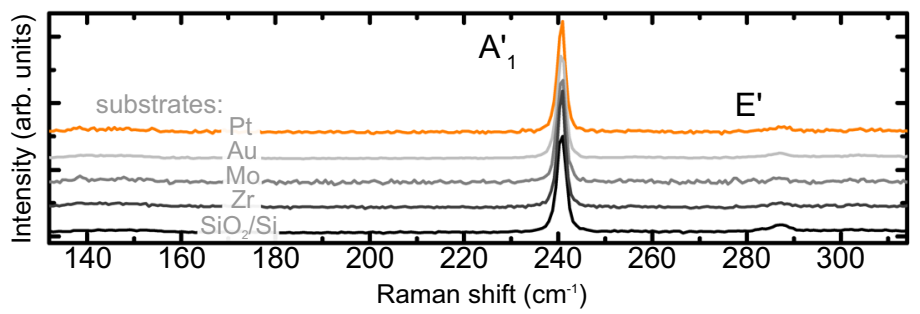

(b)
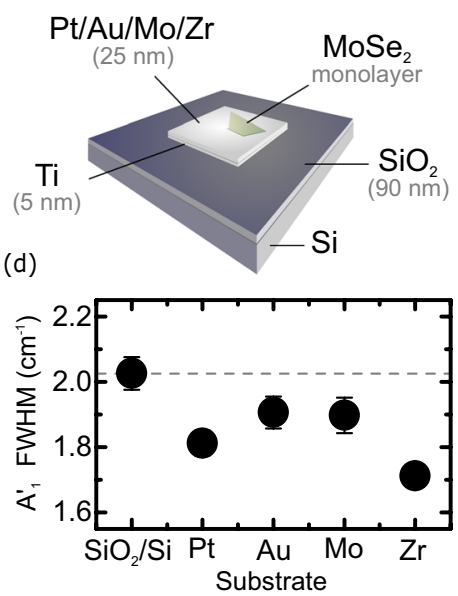

Figure 1. (a) A scheme of energy levels diagram of considered $\mathrm{MoSe}_{2} /$ metal heterostructures. The electron affinity and metal work function are denoted with $\chi$ and $\Phi$, respectively; CB and VB mark the bottom of the conduction and top of the valence bands, $E_{g}$ is the energy band gap of $\mathrm{MoSe}_{2} \mathrm{ML}$. All values are given in electronvolts (eV). (b) Schematic illustration of samples under study. (c) Room-temperature Raman scattering spectra of monolayer $\mathrm{MoSe}_{2}$ on different metallic substrates, Raman spectrum of $\mathrm{MoSe}_{2} \mathrm{ML}$ on $\mathrm{Si} / \mathrm{SiO}_{2}$ is also added as a reference. (d) The comparison of $\mathrm{A}^{\prime}{ }_{1}$ full-width-half-maximum (FWHM) on different substrates, extracted from the fitted Lorentizan functions.

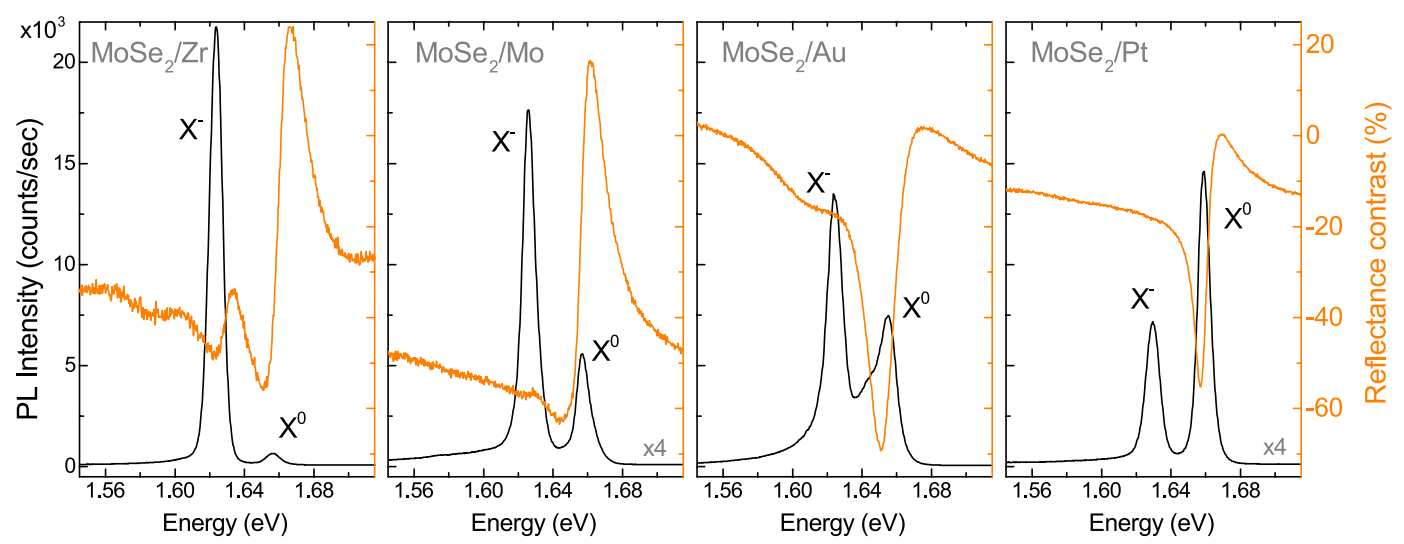

Figure 2. Photoluminescence (PL) and reflectance contrast (RC) spectra of ML MoSe $\mathrm{M}_{2}$ deposited on different metallic substrates, measured at $\mathrm{T}=5 \mathrm{~K}$. Note that the vertical scales of the PL intensity and RC are set the same for all four panels.

\section{Results}

Raman scattering spectra measured at room temperature on the studied structures are presented in Fig. 1(c). The Raman scattering spectrum of the $\mathrm{MoSe}_{2} \mathrm{ML}$ exfoliated on $\mathrm{Si} / \mathrm{SiO}_{2}$ is also shown for comparison. The spectra display two modes: an in-plane $\mathrm{E}^{\prime}$ mode at $240 \mathrm{~cm}^{-1}$ and an out-of-plane $\mathrm{A}_{1}^{\prime}$ mode at $\sim 290 \mathrm{~cm}^{-1}$. These modes are characteristic of $\mathrm{MoSe}_{2} \mathrm{MLs}^{20}$, which confirms the single-layer thickness of the investigated samples. It is well known, that the strain and disorder are great factors in shaping the properties of TMD monolayers. Their impact is well reflected in Raman scattering spectra ${ }^{21-24}$. As can be seen in Fig. 1(c), the Raman scattering spectra of $\mathrm{MoSe}_{2}$ MLs on metals and on $\mathrm{Si} / \mathrm{SiO}_{2}$ are very similar. No additional features in the energy range presented, as well as no apparent broadening of the observed phonon modes (see Fig. 1(d)) suggest that the studied MLs were not significantly affected by either the metal, on which the flakes were deposited or strain and disorder that could have been introduced into the flakes during the fabrication process. Therefore both factors are not included in further analysis of our results.

The photoluminescence (PL) spectra, shown in Fig. 2 with black lines comprise two well-separated emission lines, which are attributed to the neutral $\left(\mathrm{X}^{0} \sim 1.66 \mathrm{eV}\right)$ and the negatively charged $\left(\mathrm{X}^{-} \sim 1.63 \mathrm{eV}\right)$ excitons formed in the vicinity of the so-called A exciton at the $\mathrm{K}^{ \pm}$points of the Brillouin zone ${ }^{16,25}$. The assignment of the trion complex to the particular charge sign (positive or negative) is not straightforward. Commercially available materials used for exfoliation, are typically unintentionally doped. Moreover, the doping can vary spatially and correlates to the presence of hydrogen in underlying substrates ${ }^{26,27}$. The majority of reports on $\mathrm{MoSe}_{2}$ states unintentional $\mathrm{n}$-doping in the material ${ }^{28-30}$. Consequently, we adapt the same assumption. The arguments for 

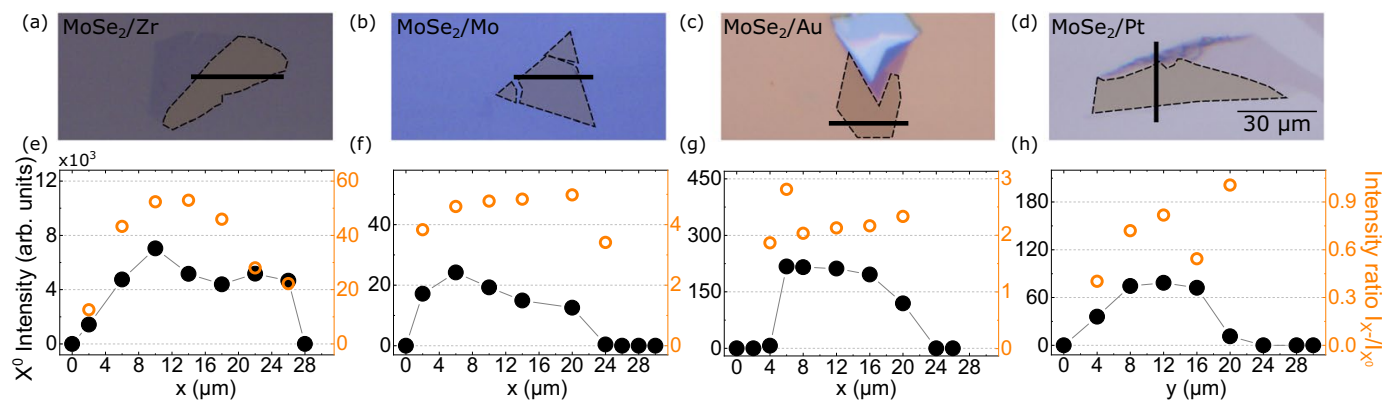

Figure 3. (a-d) Optical images of the investigated flakes. Dashed lines indicate the boundaries of $\mathrm{MoSe}_{2} \mathrm{MLs}_{\text {. }}$ $(\mathbf{e}-\mathbf{h})$ The neutral exciton $\left(\mathrm{X}^{0}\right)$ intensity accompanied with the intensity ratio of the trion $\left(\mathrm{X}^{-}\right)$to the $\mathrm{X}^{0}$ line measured at $\mathrm{T}=5 \mathrm{~K}$ on $\mathrm{MoSe}_{2}$ as a function of the position along the lines indicated in the respective images shown above.

n-type doping of the studied MLs are presented in the following section. Additionally, a third feature at around $1.65 \mathrm{eV}$ can be observed in the PL spectrum of the ML deposited on the Au substrate. No similar emission peak was reported so far for $\mathrm{MoSe}_{2}$ MLs. A possible assignment of this peak is difficult as the contribution of phonons, dark excitons and biexcitons is not very likely. By comparing the spectra (with panels arranged by increasing metal work function from the left- to the right-hand side) an obvious trend can immediately be noticed. With increasing work function of the metal, the relative intensity of the neutral excitonic line to the charged exciton line increases. For the $\mathrm{MoSe}_{2} / \mathrm{Zr}$ structure, the emission-related to the neutral exciton $\mathrm{X}^{0}$ is approx. 40 times weaker than that of the charged exciton. On the other hand, the intensities of the $\mathrm{X}^{-}$lines are about three and two times larger as compared to the $\mathrm{X}^{0}$ peaks for the $\mathrm{MoSe}_{2} / \mathrm{Mo}$ and $\mathrm{MoSe}_{2} / \mathrm{Au}$ structures, respectively. In the case of $\mathrm{MoSe}_{2} / \mathrm{Pt}$ structure, for which the metal work function is highest, the neutral exciton emission is about two times stronger as compared to the charged exciton one. An analogous effect can be recognized in the reflectance contrast (RC) results measured at $\mathrm{T}=5 \mathrm{~K}$, shown in Fig. 2 with orange lines. For three structures, i.e. $\mathrm{MoSe}_{2} / \mathrm{Zr}$, $\mathrm{MoSe}_{2} / \mathrm{Mo}$ and $\mathrm{MoSe}_{2} / \mathrm{Au}$, two resonances can be observed in the RC spectra, which are attributed to the charged and neutral excitons ${ }^{16,17,31}$. For the $\mathrm{MoSe}_{2} / \mathrm{Pt}$ stack, there is only one dip in the RC spectrum, which is ascribed to the neutral exciton.

The results described above are representative of the structures under investigation. To confirm their validity and establish homogeneity of our samples, each structure with $\mathrm{MoSe}_{2} \mathrm{ML}$ of a size approximately $20 \mu \mathrm{m}$ by $20 \mu \mathrm{m}$ (microscopic images in Fig. 3(a-d)) was measured at several spots within the flake's area. The intensity of the neutral exciton emission at each point along the cross-section of the MLs marked with black lines in Fig. 3(ad) is shown in Fig. 3(e-h). The intensity ratio of the trion $\left(\mathrm{X}^{-}\right)$and the neutral exciton $\left(\mathrm{X}^{0}\right)$ at the points is also shown in Fig. 3(e-h). Some differences in intensity ratios may result from defects of the substrate surface ${ }^{26,32}$. Moreover, a slight edge effects can be noticed, particularly in Fig. 3(e,f). The ratio decreases near the edges, which points out to the depletion of electrons in those regions. A similar effect was recently reported in $\mathrm{MoS}_{2}$ structures studied through the tip-enhanced Raman spectroscopy and was related to the edge states capturing electrons near the structure edges. ${ }^{33}$

To examine the emission spectra of studied $\mathrm{MoSe}_{2}$ MLs in more detail, we performed PL measurements over a wide temperature range from $5 \mathrm{~K}$ to $300 \mathrm{~K}$. It is known that increasing the temperature of typical MoSe $\mathrm{MLs}_{2}$ deposited on $\mathrm{Si} / \mathrm{SiO}_{2}$ leads to quickly vanishing $\mathrm{X}^{-}$emission ${ }^{16}$. Selected PL spectra are shown in Fig. 4 . In order to maintain the legibility of the plot, the spectra are displaced vertically and, if needed, multiplied by a scaling factor. Two main effects of temperature can be noticed. At low temperature, the PL spectrum of the $\mathrm{MoSe}_{2} / \mathrm{Zr}$ sample is dominated by the trion's contribution. The trion emission rapidly quenches as temperature increases and the emission can not be observed at $\mathrm{T}>200 \mathrm{~K}$. In the case of three other structures, i.e. $\mathrm{MoSe}_{2} / \mathrm{Mo}, \mathrm{MoSe}_{2} / \mathrm{Au}$ and $\mathrm{MoSe}_{2} / \mathrm{Pt}$, the $\mathrm{X}^{-}$emission disappears from the PL spectra more quickly and it can not be recognized at $\mathrm{T}>120 \mathrm{~K}$. Finally, for all the studied structures, only the $\mathrm{X}^{0}$ line is apparent in the PL spectra at $\mathrm{T}>200 \mathrm{~K}$. The $\mathrm{X}^{0}$-exciton feature shows an overall redshift consistent with the temperature evolution of the band gap ${ }^{16}$.

\section{Discussion}

The observed effects of the metallic substrates on the optical response of the $\mathrm{MoSe}_{2} \mathrm{ML}$ can be explained in terms of the corresponding doping. A schematic representation of energy levels of the studied ML and the metals used as substrates is presented in Fig. 1(a). It is important to mention that being aware of a significant effect of surrounding environment of the S-TMD ML on the magnitude of its electronic band gap $\left(E_{g}\right)^{34,35}$, we decided to implement the mean value of the optical band gap $\left(E_{g}^{\text {opt }}\right)$ of the studied MLs in the former analysis of the metal/ semiconductor junctions. Our approach results from the fact that the $E_{g}^{o p t}$ value, defined as the energy difference between the $E_{g}$ and the $\mathrm{X}^{0}$ binding energy $\left(E_{b}\right)$, is much less affected by the surrounding environment of the ML, as can be seen in Fig. 2. This indicates that the electronic band gap $\left(E_{g}\right)$ renormalization is almost completely compensated by the renormalization of the $E_{b}$ resulting in a small variation of the optical band gap $E_{g}^{o p t 36,37}$. As can be seen in Fig. 1(a), the relative position of the Fermi levels in metals with respect to the conduction band (CB) and valence band (VB) edges in the $\mathrm{MoSe}_{2} \mathrm{ML}$ changes significantly due to the variation of the metal work function. For the two metals characterised by extreme work functions, i.e. $\mathrm{Zr}$ and $\mathrm{Pt}$, their Fermi levels coincide correspondingly with the extrema of the $\mathrm{CB}$ and $\mathrm{VB}$ of the $\mathrm{MoSe}_{2} \mathrm{ML}$. This may result in the creation of metal/ 


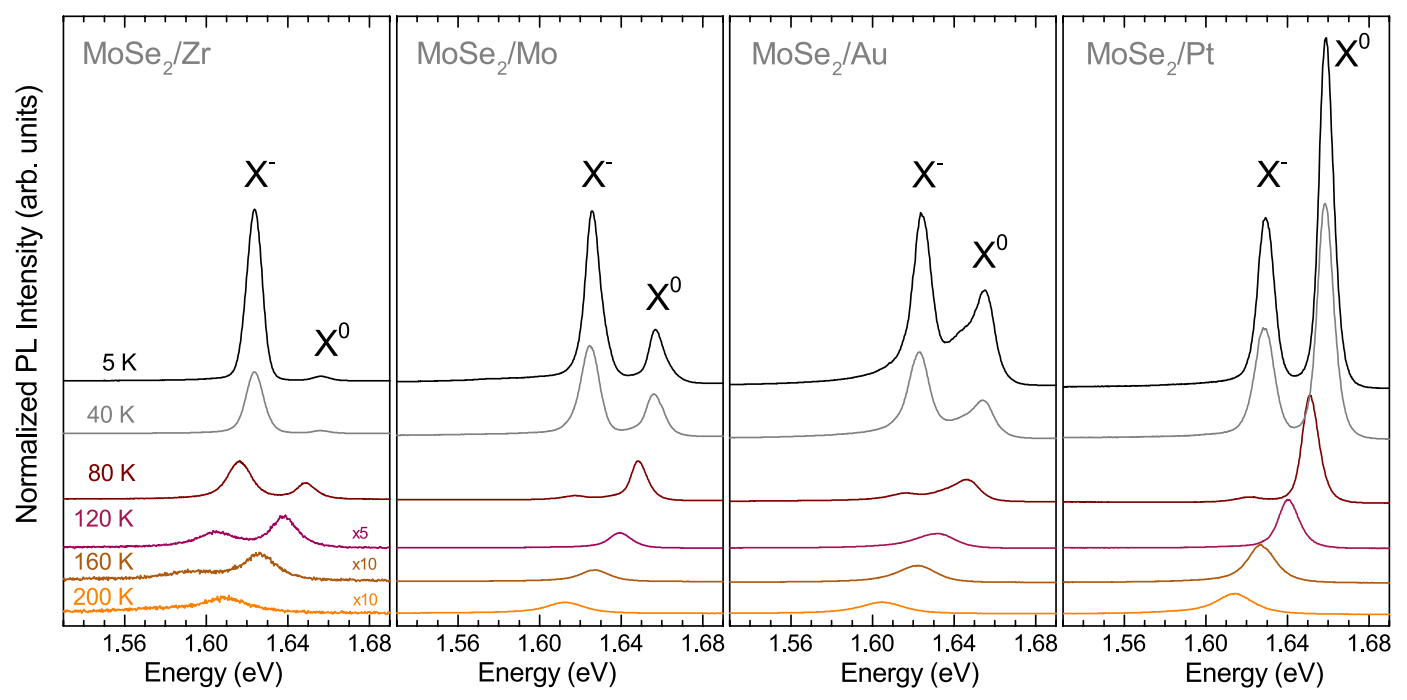

Figure 4. Temperature evolution of PL spectra of $\mathrm{MoSe}_{2} \mathrm{MLs}$ deposited on different metallic substrates. The $\mathrm{PL}$ spectra are normalized to the intensity of the $\mathrm{X}^{-}$line at $5 \mathrm{~K}$. The spectra are vertically shifted for clarity and some of them are multiplied by scaling factors in order to avoid their intersections with the neighbouring experimental curves.

semiconductor junctions, which exhibit $\mathrm{n}$ - $(\mathrm{Zr})$ or p-type $(\mathrm{Pt})$ characteristics and therefore permit to form, respectively, the negatively or positively charged excitons. Our observation is in good agreement with data that have recently been reported for TMD/metal interfaces ${ }^{38-40}$.

Let us analyse the energy of the $\mathrm{CB}$ and the VB extrema of $\mathrm{MoSe}_{2} \mathrm{ML}$ in reference to the metals' work functions. Similar energy values of $\mathrm{MoSe}_{2}$ affinity and $\mathrm{Zr}$ work function result in the band alignment. Electrons can easily transfer between the $\mathrm{MoSe}_{2} \mathrm{CB}$ and the metal surface, shifting up the Fermi level. In that case, the structure can be characterised as a Schottky barrier, which serves as an efficient electron trap. As a consequence of that band alignment, one expects that the studied ML deposited on $\mathrm{Zr}$ reveals relatively high n-type doping. This leads to the appearance of the negatively charged excitons in both the PL and RC spectra (see Fig. 2). The high doping level in the $\mathrm{MoSe}_{2} / \mathrm{Zr}$ structure results in the observation of the $\mathrm{X}^{-}$resonance in the corresponding RC spectrum measured at $\mathrm{T}=5 \mathrm{~K}$ (see Fig. 2). The $\mathrm{MoSe}_{2}$ MLs on Mo and Au substrates are less n-type doped, but still two $\mathrm{X}^{0}$ and $\mathrm{X}^{-}$resonances can be recognized in both corresponding RC and PL spectra (see Fig. 2). In those two cases (Mo and $\mathrm{Au}$ ), the Fermi energy of the metal is located within the $\mathrm{MoSe}_{2} \mathrm{ML}$ energy band gap. Assuming that the exfoliated $\mathrm{MoSe}_{2}$ crystals were intentionally undoped, their Fermi levels should be in the middle of the energy gap as in conventional semiconductors. That would amount to the energy of approx. $4.69 \mathrm{eV}$, i.e. close to the work functions of $\mathrm{Mo}(4.6 \mathrm{eV})$ and $\mathrm{Au}(5.1 \mathrm{eV})$. Consequently, it was expected that the $\mathrm{MoSe}_{2} \mathrm{MLs}$ would remain essentially undoped when placed on Mo or Au substrates, and only the neutral exciton resonance would be observed in the RC and PL spectra. As can be seen in Fig. 2, the $\mathrm{X}^{0}$ and $\mathrm{X}^{-}$transitions are apparent in both types of experiments, which strongly suggests that the exfoliated $\mathrm{MoSe}_{2}$ crystals are unintentionally $\mathrm{n}$-doped. Note that the measured PL spectra of $\mathrm{MoSe}_{2}$ deposited on $\mathrm{Zr}$, Mo, and Au substrates resemble those of typically studied $\mathrm{MoSe}_{2}$ samples on $\mathrm{Si} / \mathrm{SiO}_{2}$ substrates ${ }^{16,25,34,41}$. The spectra of the $\mathrm{MoSe}_{2} / \mathrm{Pt}$ structure show that the neutral exciton emission is more intense than the trion one. As platinum's work function falls within the VB of the investigated ML, the p-type doping in the $\mathrm{MoSe}_{2} \mathrm{ML}$ can be expected in such a case. However, as we already discussed, the $\mathrm{MoSe}_{2}$ crystals used for exfoliation were probably unintentionally n-doped. The deposition of the $\mathrm{ML}$ on the Pt substrate results in a significant decrease of the $\mathrm{X}^{-}$intensity, but does not permit to create positively charged excitons. Moreover, as it was shown in ref. ${ }^{29}$, the binding energy of the negative trion is affected by electrostatically-tuned doping level to larger extent than the binding energy of the positive trion, which may also support our attribution of the lower energy feature in the PL spectra to the negative trion (see Fig. 5(b)).

Figure $5(\mathrm{a}, \mathrm{b})$ present the trion to neutral exciton intensity ratio $\left(\mathrm{I}_{X^{-}} / \mathrm{I}_{X^{0}}\right)$ and the energy difference $\left(\Delta E_{X^{-}}=E_{X^{0}}-E_{X^{-}}\right)$between the neutral and charged exciton emission lines. Note that $\Delta E_{X^{-}}$can be defined as the binding (dissociation) energy of the charged exciton, which is the energy required to promote one of the trion's electrons to the CB edge in the limit of infinitesimally small doping ${ }^{42,43}$. As can be seen in Fig. 5 , both the intensity ratio and the trion's binding energy systematically decrease with the increase of the metal work function. The quantitative impact of the work functions on the observed changes, shown in Fig. 5(a,b), varies considerably. While the trion binding energy changes about $10 \%$ with increasing the work function, the intensity ratio decreases more than 50 times. It is important to mention that the influence of the metallic substrate on the trion binding energy is probably accompanied by the variation of the neutral exciton binding energy $\left(\Delta E_{X^{0}}\right)$, similarly as it was demonstrated for different dielectric environments of S-TMD monolayers ${ }^{44,45}$. However, a recent theoretical work ${ }^{46}$ demonstrates that the ratio of the trion to the exciton binding energy $\left(\Delta E_{X^{-}} / \Delta E_{X^{0}}\right)$ is not fixed, but changes with the environment of the ML. In consequence, we are not able to quantitatively estimate the effect of metallic substrate on the neutral exciton binding energy. 
(a)

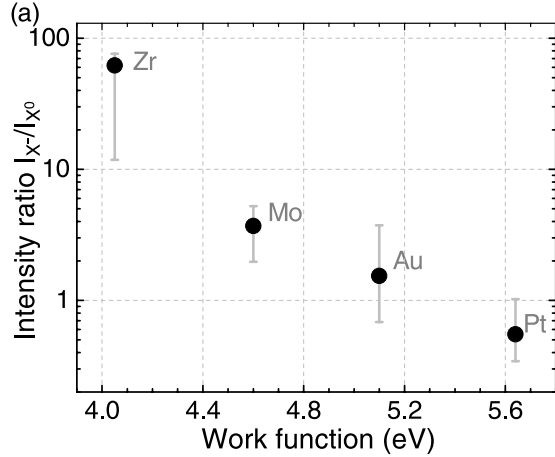

(b)

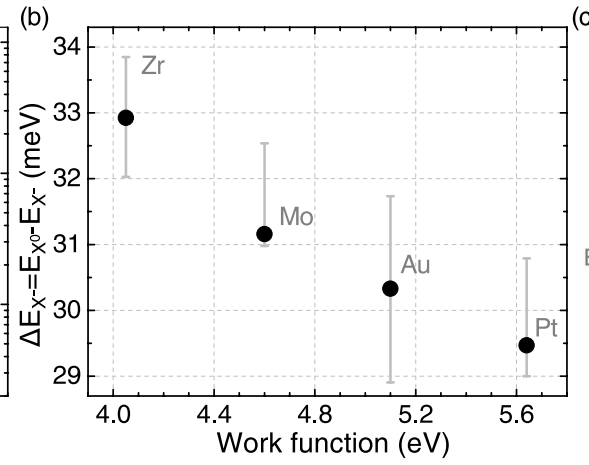

(c)

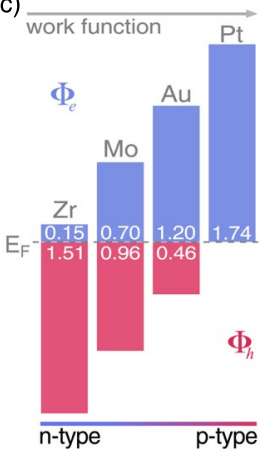

Figure 5. (a) Intensity ratio of the charged exciton to the neutral exciton line and (b) charged exciton binding energy $\left(\Delta E_{X^{-}}\right)$versus the metal work function. The grey error bars represent the deviation range of the values marked with solid circles (extracted from Fig. 2), obtained by analysing measurements from different spots within the flake's area, partially shown in Fig. 3. (c) Comparison of the calculated Schottky Barrier Heights $(\Phi)$ for selected metal/MoSe $e_{2}$ junctions.

In many practical cases, metal-semiconductor junctions can be reasonably described by a simple model relying on the Schottky Barrier Height $(\Phi)$, which is the energy, charge carriers have to overcome while being transported across the junction. The possibility of tuning $\Phi$ is highly desirable for various reasons, most of which determine the quality of electronic devices based on TMDs, especially from the viewpoint of the reduction of contact resistance ${ }^{47}$. The biggest difficulty in constructing efficient electrical contacts to TMD layers is so-called Fermi level pinning (FLP) ${ }^{48,49}$. The strength of FLP in a given semiconductor brought into contact with a set of metals of different work functions can be characterised by a slope of linear dependence fitted to the $\Phi$-versus- $\chi$ data. In our case, we neglect the contribution from this effect by assuming weak interactions between the metal and the $\mathrm{MoSe}_{2} \mathrm{ML}^{39,50,51}$. By using the Schottky-Mott model it is straightforward to calculate the Schottky Barrier Heights for various metal/semiconductor junctions:

$$
\begin{aligned}
& \Phi_{e}=\Phi-\chi \\
& \Phi_{h}=E_{i p}-\Phi
\end{aligned}
$$

in which $\Phi_{e}$ and $\Phi_{h}$ are the barrier heights for electrons and holes, respectively, $\chi$ is the semiconductor electron affinity, and $E_{i p}$ denotes the ionization potential. The obtained values are presented in Fig. 5(c). Our results show good agreement with the above analysis based on the relative alignment of the conduction and valence bands in the $\mathrm{MoSe}_{2}$ MLs and metals' work function sketched in Fig. 1. As can be appreciated in Fig. 5, the lowest Schottky barrier height for electrons of about $0.15 \mathrm{eV}$ is obtained for $\mathrm{Zr}$, while the highest one, equal to about $1.74 \mathrm{eV}$, for Pt. Interestingly, the Schottky barrier height for holes is almost 0 for Pt. These simple calculations support our conclusion based on experimental results, that the type of doping in $\mathrm{MoSe}_{2} \mathrm{ML}$ can be altered in a controlled way by placing it on metallic substrates with selected work functions.

\section{Conclusions}

We have investigated the effect of metallic (Pt, $\mathrm{Au}, \mathrm{Mo}$, or $\mathrm{Zr}$ ) substrate on the optical response of $\mathrm{MoSe}_{2} \mathrm{ML}$. It has been found that the emission intensity ratio of the charged to neutral excitons as well as the trion binding energy decrease with increasing the work function of the substrate. Our measurements reveal that the PL and RC spectra of the structure expected to exhibit the p-type characteristics $\left(\mathrm{MoSe}_{2} / \mathrm{Pt}\right)$ are dominated by the neutral exciton. When the Fermi level of metals falls inside the $\mathrm{MoSe}_{2}$ ML band gap, like for Mo and Au, both the PL and RC spectra show two resonances due to the neutral and charged excitons. On the contrary, in the structure with the metal's work function matching the bottom of the semiconductor's CB $\left(\mathrm{MoSe}_{2} / \mathrm{Zr}\right)$ strong resonances originating from the negatively charged exciton are seen in both the PL and RC spectra. We explain this effect in terms of variable doping of the $\mathrm{MoSe}_{2} \mathrm{ML}$ induced by the metal substrate. The alignment of the energy levels at the metal/semiconductor junction allows for the transfer of carriers between the layers. The presented results demonstrate a doping method of ML TMDs by appropriately selecting the metallic substrates. A versatility of standard optical experimental methods like PL and RC is demonstrated. It is shown, that they can be successfully used to check the quality and characteristics of metal/semiconductor junctions.

\section{Methods}

Metallic substrates were prepared by laser lithography and e-beam evaporation employed for patterning pieces of an $\mathrm{Si} /(90 \mathrm{~nm}) \mathrm{SiO}_{2}$ wafer with a network of slabs made of $5 \mathrm{~nm}$ thick Ti adhesion layer followed by $25 \mathrm{~nm}$ thick $\mathrm{Pt}, \mathrm{Mo}, \mathrm{Au}$, or $\mathrm{Zr}$ layer. $\mathrm{MoSe}_{2}$ MLs were prepared by all-dry PDMS-based exfoliation ${ }^{52}$ of bulk crystals purchased from HQ Graphene. The flakes of interest were first identified under an optical microscope and then subjected to atomic force microscopy and Raman spectroscopy characterisation to unambiguously determine their thicknesses and assess their overall quality. Right before transferring the flakes onto a chosen substrate, the substrate's surface was cleaned with either dry $\mathrm{CHF}_{3}$ reactive-ion-plasma $(\mathrm{Pt}, \mathrm{Au}, \mathrm{Mo})$ or wet $\mathrm{HF}$ etching $(\mathrm{Zr})$ to remove the native oxide layer and other possible contaminants. A schematic representation of the samples is shown in Fig. 1(b). To verify the credibility of the obtained results, two sets of samples were produced in the same manner. 
The investigated samples were placed on a cold finger of a continuous flow cryostat mounted on $x-y$ motorized positioners. The excitation light was focused through a 50x long-working distance objective with a 0.5 numerical aperture giving a laser spot of about $1 \mu \mathrm{m}$ diameter. The signal was collected via the same microscope objective, sent through a $0.5 \mathrm{~m}$ monochromator, and then detected by a CCD camera. The PL measurements were carried out using $\lambda=514.5 \mathrm{~nm}$ radiation from a continuous wave $\mathrm{Ar}^{+}$ion laser. The excitation power focused on the sample was kept at $\sim 50 \mu \mathrm{W}$ during all PL measurements to avoid local heating. For the RC study, a $100 \mathrm{~W}$ tungsten halogen lamp was used as a source of excitation. Light from the lamp was coupled to a multimode fiber of 50 $\mu \mathrm{m}$ core diameter, and then collimated and focused on the sample to a spot of about $4 \mu \mathrm{m}$ diameter. The RC spectra are defined as $R C(E)=\frac{R(E)-R_{0}(E)}{R(E)+R_{0}(E)} \times 100 \%$, in which $R(E)$ and $R_{0}(E)$ is the reflectance of the sample with the $\mathrm{MoSe}_{2} \mathrm{ML}$ and of the same structure without the ML, respectively. The unpolarized Raman scattering measurements were carried out in the backscattering geometry using a $\lambda=532 \mathrm{~nm} \mathrm{CW}$ diode laser. The power of light on the samples did not exceed $70 \mu \mathrm{W}$. The collected Raman signal was dispersed by a $0.75 \mathrm{~m}$ spectrometer equipped with 1800 grooves/mm gratings.

\section{Data availability}

The datasets obtained during experiments and analysis in course of manuscript preparation are available from the corresponding author on reasonable request.

Received: 4 December 2019; Accepted: 26 February 2020;

Published online: 18 March 2020

\section{References}

1. Mak, K. F., Lee, C., Hone, J., Shan, J. \& Heinz, T. F. Atomically thin $\mathrm{MoS}_{2}$ : a new direct-gap semiconductor. Physical Review Letters $105,136805(2010)$.

2. Ramasubramaniam, A. Large excitonic effects in monolayers of molybdenum and tungsten dichalcogenides. Physical Review $B$ 86, 115409 (2012).

3. Raja, A. et al. Coulomb engineering of the bandgap and excitons in two-dimensional materials. Nature Communications 8, 15251 (2017).

4. Borghardt, S. et al. Engineering of optical and electronic band gaps in transition metal dichalcogenide monolayers through external dielectric screening. Physical Review Materials 1, 054001 (2017).

5. Gupta, G., Kallatt, S. \& Majumdar, K. Direct observation of giant binding energy modulation of exciton complexes in monolayer $\mathrm{MoSe}_{2}$. Phys. Rev. B 96, 081403 (2017).

6. Steinke, C. et al. Noninvasive control of excitons in two-dimensional materials. Phys. Rev. B 96, 045431 (2017).

7. Rosner, M. et al. Two-dimensional heterojunctions from nonlocal manipulations of the interactions. Nano Letters 16, 2322-2327 (2016).

8. Kang, J. Sarkar, D. Liu, W. Jena, D. \& Banerjee, K. A computational study of metal-contacts to beyond-graphene 2D semiconductor materials. In Electron Devices Meeting (IEDM), 2012 IEEE International, 17-4 (IEEE, 2012).

9. Cakir, D. \& Peeters, F. Dependence of the electronic and transport properties of metal- $\mathrm{MoSe}_{2}$ interfaces on contact structures. Physical Review B 89, 245403 (2014).

10. Duan, X., Wang, C., Pan, A., Yu, R. \& Duan, X. Two-dimensional transition metal dichalcogenides as atomically thin semiconductors: opportunities and challenges. Chemical Society Reviews 44, 8859-8876 (2015).

11. Radisavljevic, B., Radenovic, A., Brivio, J., Giacometti, i. V. \& Kis, A. Single-layer $\operatorname{MoS}_{2}$ transistors. Nature Nanotechnology 6 , $147-150(2011)$

12. Perkins, F. K. et al. Chemical vapor sensing with monolayer $\mathrm{MoS}_{2}$. Nano Letters 13, 668-673 (2013).

13. Yuan, H. et al. Zeeman-type spin splitting controlled by an electric field. Nature Physics 9, 563-569 (2013).

14. Song, Z. et al. Tunable valley polarization and valley orbital magnetic moment hall effect in honeycomb systems with broken inversion symmetry. Scientific Reports 5 (2015).

15. Kormányos, A. et al. $\mathrm{k} \cdot \mathrm{p}$ theory for two-dimensional transition metal dichalcogenide semiconductors. 2D Materials 2, 022001 (2015).

16. Arora, A., Nogajewski, K., Molas, M., Koperski, M. \& Potemski, M. Exciton band structure in layered $\mathrm{MoSe}_{2}$ : from a monolayer to the bulk limit. Nanoscale 7, 20769-20775 (2015).

17. Koperski, M. et al. Optical properties of atomically thin transition metal dichalcogenides: observations and puzzles. Nanophotonics 6, 1289-1308 (2017).

18. Franken, P. \& Ponec, V. Ethylene adsorption on thin films of Ni, Pd, Pt, Cu, Au and Al; work function measurements. Surface Science 53, 341-350 (1975).

19. Eastman, D. Photoelectric work functions of transition, rare-earth, and noble metals. Physical Review B 2, 1 (1970).

20. Tonndorf, P. et al. Photoluminescence emission and raman response of monolayer $\mathrm{MoS}_{2}, \mathrm{MoSe}_{2}$, and $\mathrm{WSe}_{2}$. Optics Express 21, 4908-4916 (2013).

21. Liu, T. et al. Crested two-dimensional transistors. Nature nanotechnology 14, 223 (2019).

22. Mignuzzi, S. et al. Effect of disorder on raman scattering of single-layer $\mathrm{MoS}_{2}$. Physical Review B 91, 195411 (2015).

23. Gołasa, K. et al. The disorder-induced raman scattering in $\mathrm{Au} / \mathrm{MoS}_{2}$ heterostructures. Aip Advances 5, 077120 (2015).

24. Gołasa, K. et al. Optical properties of molybdenum disulfide $\left(\mathrm{MoS}_{2}\right)$. Acta Physica Polonica, A.124, 849 (2013).

25. Molas, M. et al. Brightening of dark excitons in monolayers of semiconducting transition metal dichalcogenides. $2 D$ Materials 4 , 021003 (2017).

26. Vishwanath, S. et al. Comprehensive structural and optical characterization of mbe grown $\mathrm{MoSe}_{2}$ on graphite, $\mathrm{CaF}_{2}$ and graphene. 2D Materials 2, 024007 (2015)

27. Kang, Y. \& Han, S. An origin of unintentional doping in transition metal dichalcogenides: the role of hydrogen impurities. Nanoscale 9, 4265-4271 (2017).

28. Singh, A. et al. Coherent electronic coupling in atomically thin $\mathrm{MoSe}_{2}$. Physical Review Letters 112, 216804 (2014).

29. Ross, J. S. et al. Electrical control of neutral and charged excitons in a monolayer semiconductor. Nature Communications 4, 1474 (2013).

30. Mak, K. F., He, K., Shan, J. \& Heinz, T. F. Control of valley polarization in monolayer $\mathrm{MoS}_{2}$ by optical helicity. Nature Nanotechnology 7, 494 (2012)

31. Koperski, M. et al. Orbital, spin and valley contributions to zeeman splitting of excitonic resonances in $\mathrm{MoSe}_{2}, \mathrm{WSe}_{2}$ and $\mathrm{WS}_{2}$ monolayers. 2D Materials 6, 015001, 10.1088\%2F2053-1583\%2Faae14b (2018). 
32. Wasey, A. A., Chakrabarty, S. \& Das, G. Substrate induced modulation of electronic, magnetic and chemical properties of $\mathrm{MoSe}_{2}$ monolayer. AIP Advances 4, 047107 (2014).

33. Huang, T.-X. et al. Probing the edge-related properties of atomically thin $\mathrm{MoS}_{2}$ at nanoscale. Nature Communications 10, 5544 (2019).

34. Wang, G. et al. Exciton states in monolayer $\mathrm{MoSe}_{2}$ : impact on interband transitions. 2D Materials 2, 045005 (2015).

35. Lu, J. et al. Identifying and visualizing the edge terminations of single-layer $\mathrm{MoSe}_{2}$ island epitaxially grown on au (111). ACS nano 11, 1689-1695 (2017).

36. Ugeda, M. M. et al. Giant bandgap renormalization and excitonic effects in a monolayer transition metal dichalcogenide semiconductor. Nature materials 13, 1091-1095 (2014).

37. Zhang, Q. et al. Bandgap renormalization and work function tuning in $\mathrm{MoSe}_{2} / \mathrm{hBN} / \mathrm{Ru}(0001)$ heterostructures. Nature communications $7,1-7$ (2016)

38. Liu, Y., Stradins, P. \& Wei, S.-H. Van der waals metal-semiconductor junction: Weak fermi level pinning enables effective tuning of schottky barrier. Science Advances 2, e1600069 (2016).

39. Pan, Y. et al. Interfacial properties of monolayer $\mathrm{MoSe}_{2}$-metal contacts. The Journal of Physical Chemistry C 120, 13063-13070 (2016).

40. Rosner, M. et al. Two-dimensional heterojunctions from nonlocal manipulations of the interactions. Nano Letters 16, 2322-2327 (2016).

41. Kioseoglou, G., Hanbicki, A. T., Currie, M., Friedman, A. L. \& Jonker, B. T. Optical polarization and intervalley scattering in single layers of $\mathrm{MoS}_{2}$ and $\mathrm{MoSe}_{2}$. Scientific reports 6, 25041 (2016).

42. Huard, V., Cox, R., Saminadayar, K., Arnoult, A. \& Tatarenko, S. Bound states in optical absorption of semiconductor quantum wells containing a two-dimensional electron gas. Physical Review Letters 84, 187 (2000).

43. Mak, K. F. et al. Tightly bound trions in monolayer $\mathrm{MoS}_{2}$. Nature materials 12, 207 (2013).

44. Raja, A. et al. Coulomb engineering of the bandgap and excitons in two-dimensional materials. Nature Communications 8 (2017).

45. Molas, M. R. et al. Energy spectrum of two-dimensional excitons in a nonuniform dielectric medium. Phys. Rev. Lett. 123, 136801 (2019).

46. Hichri, A., Jaziri, S. \& Goerbig, M. O. Charged excitons in two-dimensional transition metal dichalcogenides: Semiclassical calculation of berry curvature effects. Phys. Rev. B 100, 115426 (2019)

47. $\mathrm{Xu}$, S. et al. Universal low-temperature ohmic contacts for quantum transport in transition metal dichalcogenides. $2 D$ Materials 3 , 021007 (2016).

48. Das, S., Chen, H.-Y., Penumatcha, A. V. \& Appenzeller, J. High performance multilayer $\mathrm{MoS}_{2}$ transistors with scandium contacts. Nano letters 13, 100-105 (2012).

49. Bampoulis, P. et al. Defect dominated charge transport and fermi level pinning in $\mathrm{MoS}_{2} /$ metal contacts. ACS applied materials \& interfaces 9, 19278-19286 (2017).

50. Liu, Y., Stradins, P. \& Wei, S.-H. Van der Waals metal-semiconductor junction: Weak fermi level pinning enables effective tuning of Schottky barrier. Science Advances 2, e1600069 (2016).

51. Ouyang, B., Xiong, S. \& Jing, Y. Tunable phase stability and contact resistance of monolayer transition metal dichalcogenides contacts with metal. npj 2D Materials and Applications 2, 13 (2018).

52. Castellanos-Gomez, A. et al. Deterministic transfer of two-dimensional materials by all-dry viscoelastic stamping. 2D Materials 1, 011002 (2014).

\section{Acknowledgements}

The work has been supported by the National Science Centre, Poland (grant no. 2017/27/B/ST3/00205, 2017/27/N/ST3/01612, 2018/31/B/ST3/02111), the ATOMOPTO project (TEAM programme of the Foundation for Polish Science co-financed by the EU within the ERDFund), the EU Graphene Flagship project (No. 785219), the Nanofab facility of the Institut Néel, CNRS, and Ministry of Education, Youth and Sports of the Czech Republic under the project CEITEC 2020 (LQ1601).

\section{Author contributions}

M. Grzeszczyk carried out optical experiments and analysed the data. M.R. Molas, A. Bogucki and C. Faugeras supported the experiments. M. Grzeszczyk, K. Nogajewski and M. Bartoš fabricated the samples under study and performed their characterization. P. Kossacki, A. Babiński and M. Potemski contributed to data analysis. M. Potemski supervised the project. M. Grzeszczyk, M.R. Molas, K. Nogajewski, and A. Babiński wrote the manuscript with input from all the authors.

\section{Competing interests}

The authors declare no competing interests.

\section{Additional information}

Correspondence and requests for materials should be addressed to M.G.

Reprints and permissions information is available at www.nature.com/reprints.

Publisher's note Springer Nature remains neutral with regard to jurisdictional claims in published maps and institutional affiliations.

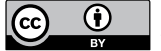

Open Access This article is licensed under a Creative Commons Attribution 4.0 International

License, which permits use, sharing, adaptation, distribution and reproduction in any medium or format, as long as you give appropriate credit to the original author(s) and the source, provide a link to the Creative Commons license, and indicate if changes were made. The images or other third party material in this article are included in the article's Creative Commons license, unless indicated otherwise in a credit line to the material. If material is not included in the article's Creative Commons license and your intended use is not permitted by statutory regulation or exceeds the permitted use, you will need to obtain permission directly from the copyright holder. To view a copy of this license, visit http://creativecommons.org/licenses/by/4.0/.

(c) The Author(s) 2020 